\title{
Search for Optical Pulsation in M82 X-2
}

\author{
G. Collura ${ }^{1}$, P. Strader ${ }^{2}$, S. R. Meeker ${ }^{1}$, P. Szypryt ${ }^{1}$, A. B. Walter ${ }^{1}$, C. Bockstiegel ${ }^{1}$, B. A. Mazin ${ }^{1}$, and T. A. Prince ${ }^{3}$ (1) \\ ${ }^{1}$ Department of Physics, University of California, Santa Barbara, California 93106, USA \\ ${ }^{2}$ Dominican School of Philosophy and Theology, 2301 Vine Street, Berkeley, CA 94708, USA \\ ${ }^{3}$ California Institute of Technology, USA \\ Received 2017 July 25; revised 2017 October 10; accepted 2017 October 13; published 2017 November 17
}

\begin{abstract}
We report on a search for optical pulsation from M82 X-2 over a range of periods. M82 X-2 is an X-ray pulsar with a $1.37 \mathrm{~s}$ average spin period and a 2.5 day sinusoidal modulation. The observations were done with the ARray Camera for Optical to Near-IR Spectrophotometry at the 200 inch Hale telescope at the Palomar Observatory. We performed $\mathrm{H}$ test and $\chi^{2}$ statistical analysis. No significant optical pulsations were found in the wavelength range of 3000-11000 $\AA$ with a pulsation period between 1.36262 and $1.37462 \mathrm{~s}$. We found an upper limit on pulsed emission in the $4000-8000 \AA$ wavelength range to be fainter than $\sim 20.5 \operatorname{mag}_{A B}$, corresponding to $\sim 23 \mu \mathrm{Jy}$.
\end{abstract}

Key words: pulsars: general - pulsars: individual (M82 X-2) - stars: neutron

\section{Introduction}

Accretion powered X-ray pulsars are magnetized neutron stars that accrete matter from a stellar companion. Ultraluminous X-rays sources (ULXs) are point sources in nearby galaxies that have luminosities that exceed the Eddington limit for a $10 M_{\odot}$ black hole. Typical luminosity ranges for ULXs are $10^{39}-10^{41} \mathrm{erg} \mathrm{s}^{-1}$ (Feng \& Soria 2011). A large fraction of ULXs are significantly variable. The low luminosity tail may encompass the brightest nearby accretion powered X-ray pulsars, with luminosities up to a few times $\sim 10^{39} \mathrm{erg} \mathrm{s}^{-1}$, such as A0538-66 (Skinner et al. 1982), SMC X-1 (Lucke et al. 1976) and GRO J1744-28 (Hyman et al. 2006). Accretion on the neutron star is mediated by a disk that couples to the neutron star's strong dipolar magnetic field $\left(10^{12}-10^{13}\right.$ Gauss). At the Alfvén radius, the flow transitions into a free fall of material along the magnetic axis. X-ray radiation can escape from the sides of the accretion column and the accretion rate can exceed the Eddington rate (Gnedin \& Sunyaev 1973; Basko \& Sunyaev 1975, 1976).

Modeling sources with luminosity greater than $10^{39} \mathrm{erg} \mathrm{s}^{-1}$ as $\sim 1-10 M_{\odot}$ stellar objects is challenging. Until recently, ULXs were interpreted as accreting black holes, either massive/stellar-mass black holes accreting above Eddington or intermediate mass black holes with masses $>100 M_{\odot}$ (Roberts 2007; Zampieri \& Roberts 2009; Feng \& Soria 2011; Liu et al. 2013; Kaaret et al. 2017). In 2014, X-ray pulsations and spin up with periodic sinusoidal modulation were observed for M82 X-2 with Chandra and NuSTAR (Bachetti et al. 2014), leading to the conclusion that it is an accreting magnetized neutron star in a binary system and not an intermediate mass black hole. After M82 X-2, two more pulsar ULXs with extremely high luminosity have been discovered: NGC 7793 P13 $\left(L_{x}=5 \times 10^{39} \mathrm{erg} \mathrm{s}^{-1}\right)$ (Fürst et al. 2016; Israel et al. 2017b) and NGC 5907 ULX1 $\left(L_{x}=2.2 \times 10^{41} \mathrm{erg} \mathrm{s}^{-1}\right)$ (Israel et al. 2017a).

\section{M82 X-2}

The X-ray luminosity of M82 X-2 is $L_{x}(3-30 \mathrm{keV})=$ $1.8 \times 10^{40} \mathrm{erg} \mathrm{s}^{-1}$, corresponding to $\sim 100 L_{\text {edd }}$ for a $1.4 M_{\odot}$ object. Explaining how such a high luminosity can be obtained is difficult, and multiple theories can constrain the strength and nature of the magnetic field based on the X-ray observations. Bachetti et al. (2014) argue for a magnetic field strength of the order of $10^{12} \mathrm{G}$ with a Roche-lobe overflow (RLOF) accretion mechanism. Christodoulou et al. (2014) also suggest a magnetic field on the order of $10^{12} \mathrm{G}$, but favor a disk-shaped wind scenario.

This value of the magnetic field is inferred from the observed spin-up rate assuming an Eddington accretion rate. The high luminosity is explained by fan beam geometry and a favorable viewing angle. Ekşi et al. (2015) argue that this value of the magnetic field is not consistent with the system being near torque equilibrium and with a fan beam geometry, and suggest instead a magnetar-like magnetic field $\left(\sim 10^{14} \mathrm{G}\right)$, and possibly even higher magnetic field strength in the multipole modes. They also favor a RLOF accretion mechanism. On the other hand, Kluźniak, King, and Lasota (Kluźniak \& Lasota 2015; King et al. 2017; King \& Lasota 2016) believe that the observed luminosity and spin-up rate indicate a much lower magnetic field $\left(<10^{9} \mathrm{G}\right)$. They also suggest that this ULX pulsar will become a millisecond pulsar in $10^{5}$ years and that accretion weakens the magnetic field, so that these objects spend a relatively short fraction of their ULX phase emitting detectable pulses. As a consequence, the authors suggest that most, if not all, of the known ULX sources might have neutron star accretors.

Other models favor dipole magnetic fields of $\sim 10^{12} \mathrm{G}$ but strong multipolar magnetic fields $\left(\sim 10^{14} \mathrm{G}\right.$; Tong 2015 ; Chen 2017; Israel et al. 2017a). Mushtukov et al. (2015) model the accretion and the radiation of energy through the walls of the accretion column. Lyutikov (2014) argues that, for highly super-Eddington sources, such a mechanism is not efficient and accretion happens in a new regime, through an optically thick accretion curtain.

Fragos et al. (2015) used binary stellar evolution and a mass transfer code to constrain the characteristic accretion: they find that the donor star has to be hydrogen rich; the mass transfer is nonconservative and efficiency $\lesssim 0.1$. They also constrain the age of the binary system, and the mass of the neutron star progenitor and of the donor.

There is no agreement about the accretion and emission mechanisms, and the characteristics of M82 X-2 and its magnetic field strength and structure are not yet well explained. 
More observations are needed, especially in different bands, to understand ULX accretion powered X-ray pulsars.

\section{ARray Camera for Optical to Near-IR Spectrophotometry (ARCONS)}

ARCONS (Mazin et al. 2013) is a 2024 pixel Microwave Kinetic Inductance Detectors (MKIDs; Day et al. 2003; Mazin et al. 2012) instrument. ARCONS detects individual photons with a $2 \mu$ s time resolution and with an energy resolution of $R=E / \Delta E \sim 8$ at $4000 \AA$. It has a passband of $3000-11000 \AA$ A. The instrument was deployed at the Coudé focus of the 200 inch Hale Telescope at Palomar Observatory. Even though other high-resolution optical instruments such as OPTIMA (Straubmeier et al. 2001), BVIT (Welsh et al. 2012), or Aqueye + and Iqueye (Naletto et al. 2009; Zampieri et al. 2015) can achieve time resolutions of the order of tens of nanoseconds to hundreds of picoseconds, ARCONS is unique in that it is an Integral Field Unit, permitting it to obtain the spectrum of the observed object without the need for filters. ARCONS has been used to search for pulsations in the millisecond pulsar PSR J0337+1715 (Strader et al. 2016).

\section{Observations and Analysis}

We observed M82 X-2 from the Cloud Focus of the Palomar 200 inch Hale telescope for 30 minutes on the night of 2014 October 21st. The observation of M82 X-2 was done in the five mirror configuration due to the decl. of M82. Seeing during the observation was below 1"!2. The ARCONS data reduction pipeline was used to extract photon lists from the raw data files (van Eyken et al. 2015). Each photon has an associated wavelength and timestamp. Optical path flat field calibration weights were not applied because they were not taken in the five mirror configuration but only in the three mirror one. As a consequence, the absolute measurement of flux is not completely reliable. We estimate that the deviation from the real flux is at most $5 \%$. However, the flux has been calibrated against a five-minute observation of the Crab pulsar done with ARCONS in the five mirror configuration immediately before the M82 X-2 observation. Since no PSF was available in the field of view, we used a finder star that was observed before pointing the telescope at M82 X-2 to determine the optimal aperture. The aperture radius was set to two pixels, roughly 0.9 arcsec, corresponding standard deviation of the Gaussian PSF fit of the finder star.

Since ARCONS was fixed at the Cloud Focus, the field of view rotates with respect to the detector. The telescope was moved periodically to ensure that the target stayed on the detector and in highly filled regions of the MKID array. The segments of observation when the telescope was moved were removed. We then tracked the target during rotation of the field of view and the telescope moves. While the center of rotation (optical axis) is unknown, rotation about an arbitrary point can be decomposed into an equal rotation about the center of the image and a translation. We first divided the 30 minute observation into $30 \mathrm{~s}$ long frames, and then aligned each frame to the first. We first accounted for the known field rotation of $15^{\circ}$ per hour. We then upsampled the frames while conserving the total flux, and aligned them to the first frame by finding the translation that minimizes the

$$
\chi^{2}=\sum_{i=0}^{n_{\mathrm{pix}}} \frac{\left(x_{i}-y_{i}\right)^{2}}{\sqrt{\left(x_{i}+y_{i}\right)^{2}}}
$$

where $x_{i}$ is the number of photons in the $i$ th pixel of the reference image (the first frame), $y_{i}$ is the number of counts in the ith pixel of the image to align after rotation and translation, and the denominator is $\sigma^{2}$, which takes into account both the error of the reference image and that of the target image. The translation accounts for both the fact that that telescope moves and the fact that the rotation center does not correspond to the center of the array. The final alignment is accurate to approximately 0.5 arcsec.

A photon list was then generated by applying an aperture of roughly 0.9 arcsec in radius at the location of X2.

We used the pulsar timing software package TEMPO ${ }^{4}$ to barycenter the photons and fold them. Since the period at the time of the observation is not known, we did a blind search between $1.36262 \mathrm{~s}$ and $1.37462 \mathrm{~s}$ with $50 \mu$ s intervals. The range of the search was determined by looking at the period in Bachetti et al. (2014) and calculating what the period would be on the observing date for the minimum and maximum $\dot{P}$ and then increased that range by a factor of four to account for unexpected behavior of the target object. The search was performed on a fine enough grid so that a period mismatch would not create appreciable smearing in the final light curve.

\section{Results}

After making the list of photons with wavelengths and barycentered time stamps, we applied statistical tests to find periodicity with different wavelength cuts and folding periods. Two factors play a role in the choice of wavelength: on one hand, the signal-to-noise ratio in the infrared is likely lower because the ARCONS detector begins to receive false photon events at longer wavelengths; on the other hand, the extinction due to dust in the galaxy is higher at bluer wavelengths. The choice of wavelength is a compromise between these two effects. We evaluated four different wavelength cuts: $4000-5500 \AA, \quad 8000-11000 \AA$, $3000-11000 \AA$, and 4000-8000 $\mathrm{A}$.

We applied a $\chi^{2}$ test for deviation from a flat line and also the $H$ test (de Jager et al. 1989) to test for periodicity at different periods and wavelength ranges. A flat line is the expected light curve if there is no pulsed emission. In the $H$ test, higher $H$ values indicate more significant periodicity.

The results for both the $H$ test and the $\chi^{2}$ test are summarized in Table 1. For each wavelength cut, we report the maximum $H$ value, the false positive probability (FPP) corresponding to it and the period at which the $H$ value is maximum. In the three rightmost columns, there are the maximum reduced $\chi^{2}$ and the corresponding FPP and period.

Since we searched over many different periods to calculate the FPP of the result, we needed to take into account the number of independent periods, often referred to as independent Fourier spacings (IFS), in our statistics. The number of IFSs for the $H$ value calculation is given by $x=T\left(\frac{1}{t_{1}}-\frac{1}{t_{2}}\right)$, where $T$ is the duration of the observation and $\left[\frac{1}{t_{1}}, \frac{1}{t_{2}}\right]$ is the frequency interval of the search. For our analysis, we have

\footnotetext{
4 http://www.atnf.csiro.au/research/pulsar/tempo/
} 
Table 1

Statistical Results of the Search of Different Wavelength Ranges over a Period Range of $12 \mathrm{~ms}$

\begin{tabular}{lrllccc}
\hline \hline & $H$ & Period & $p$-value & Reduced $\chi^{2}$ & Period \\
\hline $4000-5500 \AA$ & 13.6 & $1.36782 \mathrm{~s}$ & 0.05 & 2.6 & $1.36387 \mathrm{~s}$ & 0.7 \\
$8000-11000 \AA$ & 8.6 & $1.36417 \mathrm{~s}$ & 0.4 & 2.7 & $1.37237 \mathrm{~s}$ \\
$3000-11000 \AA$ & 9.5 & $1.37272 \mathrm{~s}$ & 0.28 & 2.5 & $1.37172 \mathrm{~s}$ \\
$4000-8000 \AA$ & 14.5 & $1.36347 \mathrm{~s}$ & 0.037 & 3.3 & $1.36357 \mathrm{~s}$ \\
\hline
\end{tabular}

Note. The most significant result has an FPP of $3.7 \%$ and a low statistical significance of $2 \sigma$.

$T=1800 \mathrm{~s}, t_{1}=1.36262 \mathrm{~s}$, and $t_{2}=1.37462 \mathrm{~s}$. This gives $\sim 12$ IFS. In the case of $\chi^{2}$, the number of IFS is $\sim 120$ because the bin size is one-tenth of the period.

In reality, the FPPs for the $H$ value are lower limits, due to the fact that, even if there are only 12 IFS, we scanned $12 \mathrm{~ms}$ at $50 \mu \mathrm{s}$ intervals, making 240 periods. This oversampling increases the FPP by a factor that decreases with the number of IFSs scanned and increases with the $H$ value (Buccheri \& de Jager 1989). This factor is estimated using simulations for up to three IFSs in de Jager et al. (1989).

The binned light curve, folded at the $P=1.36357 \mathrm{~s}$, corresponding to the maximum $\chi^{2}$ in the $4000-8000 \AA$ range, is shown in Figure 1. For this light curve, we get $\chi^{2}=29.7$ with nine degrees of freedom, which corresponds to a reduced $\chi_{\nu}^{2}$ of 3.3. The FPP for this result is $6 \%$, indicating a significance of pulsed emission of only $1.9 \sigma$.

The highest $H$ metric is 14.5 , and the corresponding period is $1.36347 \mathrm{~s}$ in the $4000-8000 \AA$ wavelength range. It gives an FPP of $3.7 \%$, also indicating a significance of pulsed emission of $2 \sigma$. The results indicate no statistically significant detection of pulsed emission.

The $H$ test also gives the number of harmonics needed to maximize the $H$ value. The number of Fourier modes that maximizes the $H$ value for our light curve is three. We constructed a model with the first three harmonics to simulate photon lists and estimate the $95 \%$ confidence upper limit on the pulsed optical emission. The model with $m$ harmonics has the form

$$
f_{m}(\theta)=\left[1+2 \sum_{k=1}^{m}\left(a_{k} \sin k \theta+b_{k} \cos k \theta\right)\right] / 2 \pi,
$$

where the coefficients $a_{k}$ and $b_{k}$ are given by

$$
\begin{aligned}
& a_{k}=1 / n \sum_{i=1}^{n} \sin k \theta_{i} \\
& b_{k}=1 / n \sum_{i=1}^{n} \cos k \theta_{i},
\end{aligned}
$$

where $\theta_{i}$ is the phase of the $i$ th photon. We generated 1000 simulated photon lists with the same number of photons as the observation, $\sim 370,000$. In each iteration, a fraction of the photon phases were randomly sampled from a uniform probability distribution and the rest where randomly sampled from a probability distribution of the form of this model with $m=3$, the latter photon phases being the ones from the source. The fraction of photons coming from the source was then increased and the fraction coming from the background was decreased keeping the total number of photons constant, until the value of the $H$ metric was 14.5 or higher for at least $95 \%$ of the trials. Since ARCONS is a photon counting instrument, the

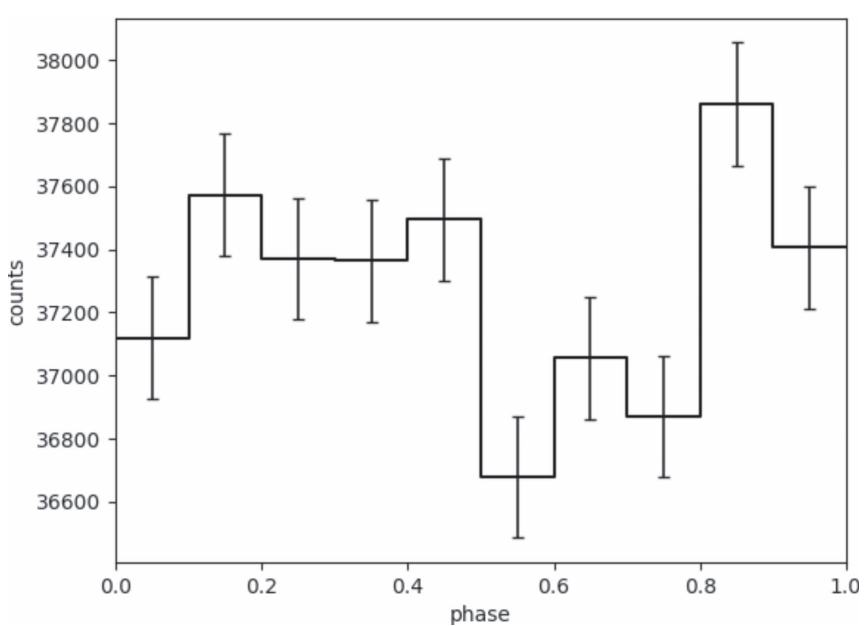

Figure 1. Light curve of M82 X-2 in the band 4000-8000 $\AA$ with a folding period of $1.36357 \mathrm{~s}$. The error bars are the square root of the total number of counts in each bin.

wavelength of the photons does not play a role in determining the fraction of photons that gives the desired value of the $H$ metric, so we did not make any assumption on the spectrum of the source in this phase.

We found that, in order to have a $95 \%$ probability to observe a light curve with an $H$ metric of 14.5 or higher, the number of photons from the pulsed emission would need to be at least $\sim 1 \%$ of the total photons. The corresponding magnitude depends on the spectrum of the source. If all the photons have a wavelength of $4000 \AA$, the source is $4.5 \mathrm{mag}$ fainter than the background, and it is 5.2 if all the photons come from a monochromatic source at $8000 \AA$. It follows that the pulsed emission is at least 4.5 mag fainter than the background. The $A B$ magnitude of the galaxy background is 16.1 , calculated by measuring the total flux in the aperture and comparing it to that of the Crab pulsar, which has a known spectrum (Nasuti et al. 1996), setting the $95 \%$ confidence upper limit on the brightness of the pulsed emission at $\sim 20.5 \mathrm{mag}_{A B}$ in the 4000-8000 А wavelength range, corresponding to a flux of $\sim 23 \mu \mathrm{Jy}$. As a reference, the same flux corresponds to a $\mathrm{V}$-band magnitude of 20.5 .

\section{Conclusions}

We presented a search for optical pulsations in the ULX pulsar M82 X-2. We observed the target for 30 minutes and performed $H$ tests and $\chi^{2}$ tests on four different wavelength ranges, looking for pulsations in a period range of $12 \mathrm{~ms}$. No statistically significant pulsations were found. We put a $95 \%$ confidence upper limit for pulsed emission at 4.5 mag fainter than the emission from the background galaxy, which sets the limit at $\sim 20.5 \mathrm{mag}_{A B}$, corresponding to $\sim 23 \mu \mathrm{Jy}$, in the 
4000-8000 ̊ wavelength range at a pulse period of $1.36347 \mathrm{~s}$. Future work can be done increasing the observing time or observing at redder wavelengths to reduce the dust extinction, for example, using the Dark-speckle Near-IR Energy-resolved Superconducting Spectrophotometer (DARKNESS) MKIDs instrument that is optimized to work in the $800-1400 \mathrm{~nm}$ wavelength band (Meeker et al. 2015).

This material is based upon work supported by the National Science Foundation under grant No. AST-1411613. Observations were made using the Palomar 200 inch Hale Telescope of the Caltech Optical Observatories. We are pleased to acknowledge the Palomar Observatory staff for their enthusiastic and excellent support.

\section{ORCID iDs}

T. A. Prince ำ https://orcid.org/0000-0002-8850-3627

\section{References}

Bachetti, M., Harrison, F. A., Walton, D. J., et al. 2014, Natur, 514, 202 Basko, M. M., \& Sunyaev, R. A. 1975, A\&A, 42, 311

Basko, M. M., \& Sunyaev, R. A. 1976, MNRAS, 175, 395

Buccheri, R., \& de Jager, O. C. 1989, in NATO Advanced Science Institutes (ASI) Series C, Vol. 262, ed. H. Ögelman \& E. P. J. van den Heuvel (New York: Kluwer), 95

Chen, W.-C. 2017, MNRAS, 465, L6

Christodoulou, D. M., Laycock, S. G. T., \& Kazanas, D. 2014, arXiv: 1411.5434

Day, P. K., LeDuc, H. G., Mazin, B. A., Vayonakis, A., \& Zmuidzinas, J. 2003, Natur, 425, 817

de Jager, O. C., Raubenheimer, B. C., \& Swanepoel, J. W. H. 1989, A\&A, 221,180
Ekşi, K. Y., Andaç, I., Çıkıntoğlu, S., et al. 2015, MNRAS, 448, L40

Feng, H., \& Soria, R. 2011, NewAR, 55, 166

Fragos, T., Linden, T., Kalogera, V., \& Sklias, P. 2015, ApJL, 802, L5

Fürst, F., Walton, D. J., Harrison, F. A., et al. 2016, ApJL, 831, L14

Gnedin, Y. N., \& Sunyaev, R. A. 1973, A\&A, 25, 233

Hyman, S. D., Lazio, T. J. W., Roy, S., et al. 2006, ApJ, 639, 348

Israel, G. L., Belfiore, A., Stella, L., et al. 2017a, Sci, 355, 817

Israel, G. L., Papitto, A., Esposito, P., et al. 2017b, MNRAS, 466, L48

Kaaret, P., Feng, H., \& Roberts, T. P. 2017, ARA\&A, 55, 303

King, A., \& Lasota, J.-P. 2016, MNRAS, 458, L10

King, A., Lasota, J.-P., \& Kluzniak, W. 2017, MNRAS, 468, L59

Kluźniak, W., \& Lasota, J.-P. 2015, MNRAS, 448, L43

Liu, J., Bregman, J. N., Bai, Y., Justham, S., \& Crowther, P. 2013, Natur, 503, 500

Lucke, R., Yentis, D., Friedman, H., Fritz, G., \& Shulman, S. 1976, ApJL, 206, L25

Lyutikov, M. 2014, arXiv:1410.8745

Mazin, B. A., Bumble, B., Meeker, S. R., et al. 2012, OExpr, 20, 1503

Mazin, B. A., Meeker, S. R., Strader, M. J., et al. 2013, PASP, 125, 1348

Meeker, S. R., Mazin, B. A., Jensen-Clem, R., et al. 2015, in Adaptive Optics for Extremely Large Telescopes 4, ed. D. Gavel et al. (Santa Cruz, CA: University of California Center for Adaptive Optics), E75

Mushtukov, A. A., Suleimanov, V. F., Tsygankov, S. S., \& Poutanen, J. 2015, MNRAS, 454, 2539

Naletto, G., Barbieri, C., Occhipinti, T., et al. 2009, A\&A, 508, 531

Nasuti, E. P., Mignani, R., Caraveo, P. A., \& Bignami, G. F. 1996, Msngr, 83,37

Roberts, T. P. 2007, Ap\&SS, 311, 203

Skinner, G. K., Bedford, D. K., Elsner, R. F., et al. 1982, Natur, 297, 568

Strader, M. J., Archibald, A. M., Meeker, S. R., et al. 2016, MNRAS, 459, 427

Straubmeier, C., Kanbach, G., \& Schrey, F. 2001, ExA, 11, 157

Tong, H. 2015, AN, 336, 835

van Eyken, J. C., Strader, M. J., Walter, A. B., et al. 2015, ApJS, 219, 14

Welsh, B., Anderson, D., McPhate, J., et al. 2012, in IAU Symp. 285, New Horizons in Time Domain Astronomy, ed. E. Griffin, R. Hanisch, \& R. Seaman (Cambridge: Cambridge Univ. Press), 99

Zampieri, L., Naletto, G., Barbieri, C., et al. 2015, Proc. SPIE, 9504, 95040C Zampieri, L., \& Roberts, T. P. 2009, MNRAS, 400, 677 